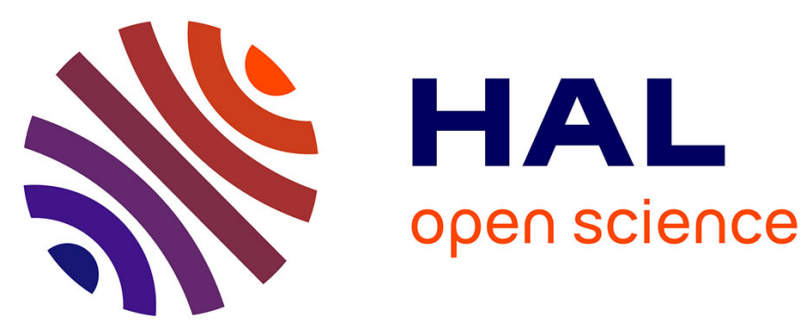

\title{
A new hybrid recursive regularised Bhatnagar-Gross-Krook collision model for Lattice Boltzmann method-based large eddy simulation
}

Jérôme Jacob, Orestis Malaspinas, Pierre Sagaut

\section{- To cite this version:}

Jérôme Jacob, Orestis Malaspinas, Pierre Sagaut. A new hybrid recursive regularised BhatnagarGross-Krook collision model for Lattice Boltzmann method-based large eddy simulation. Journal of Turbulence, 2018, pp.1 - 26. 10.1080/14685248.2018.1540879 . hal-02114308

\author{
HAL Id: hal-02114308 \\ https://hal.science/hal-02114308
}

Submitted on 29 Apr 2019

HAL is a multi-disciplinary open access archive for the deposit and dissemination of scientific research documents, whether they are published or not. The documents may come from teaching and research institutions in France or abroad, or from public or private research centers.
L'archive ouverte pluridisciplinaire HAL, est destinée au dépôt et à la diffusion de documents scientifiques de niveau recherche, publiés ou non, émanant des établissements d'enseignement et de recherche français ou étrangers, des laboratoires publics ou privés. 


\title{
A new hybrid recursive regularised Bhatnagar-Gross-Krook collision model for Lattice Boltzmann method-based large eddy simulation
}

\author{
Jérôme Jacob (10 ${ }^{\mathrm{a}}$, Orestis Malaspinas (10 ${ }^{\mathrm{b}}$ and Pierre Sagaut (1D ${ }^{\mathrm{a}}$ \\ ${ }^{a}$ Centrale Marseille, Aix Marseille University, CNRS, Marseille, M2P2, France; bepartment of Computer \\ Science, University of Geneva, Carouge, Switzerland
}

\begin{abstract}
A new Lattice Boltzmann collision model for large eddy simulation (LES) of weakly compressible flows is proposed. This model, referred to as the Hybrid Recursive Regularised Bhatnagar-Gross-Krook (HRR-BGK) model, is based on a modification of previously existing regularised collision models defined with the BGK Lattice Boltzmann method (LBM) framework. By hybridising the computation of the velocity gradient with an adequate Finite Difference scheme when reconstructing the non-equilibrium parts of the distribution function, a hyperviscosity term is introduced in the momentum equation, whose amplitude can be explicitly tuned via a weighting parameter. A dynamic version of the HRR-BGK is also proposed, in which the control parameter is tuned at each grid point and each time step in order to recover an arbitrarily fixed total dissipation. This new collision model is assessed for both explicit and implicit LES considering the flow around a circular cylinder at $\mathrm{Re}=3900$. The dynamic HRRBGK is observed to yield very accurate results when equipped with Vreman's subgrid model to compute the target dissipation.
\end{abstract}

\section{KEYWORDS}

Large eddy simulation; Lattice Boltzmann method; circular cylinder Reynolds 3900

\section{Introduction}

Large eddy simulation (LES) is now a mature simulation technique for high-resolution unsteady simulation of turbulent flows [1-3]. This technique, which is under development since the early 1960s, is now implemented in almost all computational fluid dynamics (CFD) tools, including commercial software. Among the key issues faced when developing LES-based simulation tools, one must cite the development of accurate models for subgrid scales and adequate numerical schemes that guarantee numerical stability whose induced dissipation does not overwhelm the effect of physical subgrid models. To this end, a huge amount of subgrid models and numerical methods have been proposed since the 1980s. This issue is far from being a trivial one, since it is now accepted that the discretisation errors and the subgrid models are implicitly coupled in a genuinely nonlinear way

CONTACT Jérôme Jacob jerome.jacob@univ-amu.fr E M2P2 UMR7340, Centrale Marseille Plot 6, 38 re Joliot-Curie 13451 Marseille, France 
which escapes a full mathematical analysis. Therefore, finding an optimised pair (numerical scheme and subgrid model) is a very difficult task, since it has been observed that some partial error cancellation may occur, leading to: (i) a kind of super-convergence of LES results (an unexpected high accuracy is obtained in such a case since modelling errors and numerical errors partially balance each other) and (ii) the counter-intuitive result that in some cases an increase of the order of the numerical scheme or grid refinement may lead to a decrease of the global accuracy of the results [4-6]. A promising way to solve this problem is to develop stabilised numerical schemes whose leading error terms mimic explicit subgrid models, leading to the implicit large eddy simulation (ILES) approach [7]. Using such a scheme, one may expect to guarantee numerical robustness and physical accuracy at the same time in a better controlled way.

LES has been implemented within the Lattice Boltzmann method (LBM) framework [8-12] since the 1990s [13-26], and both classical LES and ILES approaches have been proposed. The most common way to incorporate an explicit subgrid viscosity model is to modify the relaxation time in the collision model in order to recover the targeted effective total viscosity, defined as the sum of the molecular viscosity of the fluid and the subgrid viscosity. But the need to develop stabilised LBM schemes to handle high Reynolds number flows has also led to the definition of collision models with some ILES capabilities, such as Entropic methods [27-30], Cascaded models [31-33] and regularised methods [34-39]. While it has been observed in numerical experiments that these methods may lead to satisfactory results in turbulent flow simulations without adding an explicit subgrid model, the direct link between their build-in dissipation and the physical subgrid one is not fully understood.

This paper proposes a new improved recursive regularised LBM method based on the hybridisation of the computation of the velocity gradients when evaluating the coefficients of the reconstruction of the regularised non-equilibrium component of distribution functions, leading to the definition of an Hybrid Recursive Regularised Bhatnagar-Gross-Krook (HRR-BGK) model. In this new model, the numerical stabilisation can be explicitly tuned in order to recover a perfectly controlled amount of local dissipation. To get an accurate ILES method, the free parameter appearing in this new collision kernel can be set dynamically at each grid point and time step to a value which allow to recover exactly the same dissipation as the one provided by any classical LES subgrid model, according to an explicit formula given below. The scope of the paper is restricted to low-Mach number athermal flows.

This paper is organised as follows. The basic regularised BGK collision model used to develop the new collision scheme is reminded in Section 2. The new dynamic RR-BGK collision kernel is then discussed in Section 3. The analysis of its build-in dissipation and the bridge with ILES are presented in Section 4. Key features of the Vreman's subgrid model, which is used in this paper for explicit LES and tuning of the ILES simulations are reminded in Section 5. The approach is then assessed considering the turbulent flow at $\operatorname{Re}=3900$, which is a well-documented validation case. This test case is selected because it allows for a wall-resolving simulation without wall model in a flow configuration in which the subgrid model plays a very important role. The decoupling from the effects of a wall model is an important point here, since wall models may have a deeper impact on the solution than the subgrid model, masking the effect of the later. Here, the subgrid closure is known to have a leading effect on the development of the separated shear layers and the formation 
of the cylinder wake. It is worth noting that this is not the case considering plane channel flows on LBM grids. As a matter of fact, wall-resolving LES of plane channel flow would be based on a uniform grid with $\Delta x^{+}=\Delta y^{+}=\Delta z^{+}=1-2$ near the wall, which is finer than classical DNS grid resolution requirement, yielding a poor interest for the assessment of the present subgrid closure approach.

\section{Recursive regularised BGK LBM}

The Boltzmann equation discretised in the velocity space reads

$$
\partial_{t} f_{i}(\boldsymbol{x}, t)+\left(\boldsymbol{\xi}_{i} \cdot \nabla\right) f_{i}(\boldsymbol{x}, t)=\Omega_{i}
$$

where $\left\{\boldsymbol{\xi}_{i}\right\}_{i=0}^{q-1}$ is the discrete velocity set, $\Omega_{i}$ is the collision operator. The most widely used collision operator in the lattice Boltzmann community is the BGK model, where $\Omega$ is a relaxation towards a polynomial approximation of the Maxwell-Boltzmann distribution equilibrium, noted $f_{i}^{(0)}$

$$
\Omega=-\frac{1}{\tau}\left(f_{i}-f_{i}^{(0)}\right)
$$

where $f_{i}^{(0)}$ is the equilibrium distribution function

$$
f_{i}^{(0)}=w_{i} \rho\left(1+\frac{\xi_{i} \cdot \boldsymbol{u}}{c_{s}^{2}}+\frac{1}{2 c_{s}^{4}} \mathcal{H}_{i}^{(2)}: \boldsymbol{u} \boldsymbol{u}\right),
$$

with $c_{s}$ the speed of sound (which is a constant) and $\mathcal{H}_{i}^{(2)}=\boldsymbol{\xi}_{i} \boldsymbol{\xi}_{i}-c_{s}^{2} \boldsymbol{I}$ ( $\boldsymbol{I}$ is the identity matrix). This model is accurate for low-Mach number, weakly compressible athermal flows. It suffers from a lack of numerical stability in high Reynolds number flows. Therefore several successful attempts have been made to make it more stable. Among these the regularised approach (see $[35,36])$ is particularly appealing due to its simplicity. The collision operator of this model reads

$$
\Omega_{i}=-\frac{1}{\tau} f_{i}^{(1)}
$$

where

$$
f_{i}^{(1)}=-\frac{1}{2 c_{s}^{4}} \mathcal{H}_{i}^{(2)}: \boldsymbol{P}^{(1)},
$$

with $\boldsymbol{P}^{(1)}=\sum_{i=0}^{q-1} \mathcal{H}_{i}^{(2)}\left(f_{i}-f_{i}^{(0)}\right)$ is the deviatoric stress tensor.

In Ref. [37], a recursive procedure is introduced to add higher order terms to make the regularisation model even more stable, which was later used and generalised in Refs. $[34,38]$. It is based on the relation between the equilibrium and non-equilibrium Hermite coefficients of the distribution function $f_{i}$. By expanding $f_{i}$ in Hermite polynomials up to an order $N$ (see [11]) one gets

$$
f_{i}=w_{i} \sum_{n=0}^{N} \frac{1}{c_{s}^{2 n} n !} \mathcal{H}_{i}^{(n)}: \boldsymbol{a} .
$$


Then by using the standard Chapman-Enskog expansion [40], one can decompose $f_{i}$ into two parts

$$
f_{i}=f_{i}^{(0)}+f_{i}^{(1)}
$$

where $f_{i}^{(0)}$ is the equilibrium distribution function and $f_{i}^{(1)}$ is the off-equilibrium distribution function with $f_{i}^{(0)} \gg f_{i}^{(1)}$. These two distribution functions can also be expanded in Hermite polynomials

$$
\begin{aligned}
f_{i}^{(0)} & =w_{i} \sum_{n=0}^{N} \frac{1}{c_{s}^{2 n} n !} \mathcal{H}_{i}^{(n)}: \boldsymbol{a}_{0}^{(n)}, \\
f_{i}^{(1)} & =w_{i} \sum_{n=0}^{N} \frac{1}{c_{s}^{2 n} n !} \mathcal{H}_{i}^{(n)}: \boldsymbol{a}_{1}^{(n)},
\end{aligned}
$$

where $\boldsymbol{a}_{0}^{(n)}$ and $\boldsymbol{a}_{1}^{(n)}$ are the Hermite coefficients of the equilibrium and off-equilibrium distributions respectively,

$$
\begin{aligned}
& \boldsymbol{a}_{0}^{(n)}=\sum_{i=0}^{q-1} \mathcal{H}_{i}^{(n)} f_{i}^{(0)}, \\
& \boldsymbol{a}_{1}^{(n)}=\sum_{i=0}^{q-1} \mathcal{H}_{i}^{(n)} f_{i}^{(1)} .
\end{aligned}
$$

In the athermal case, the equilibrium Hermite coefficients of order $n$ are given by

$$
\begin{gathered}
\boldsymbol{a}_{0}^{(n)}=\boldsymbol{a}_{0}^{(n-1)} \boldsymbol{u}, \text { with } \\
a_{0}^{(0)}=\rho .
\end{gathered}
$$

Using the Chapman-Enskog expansion as in [37], one asymptotically first recovers the weakly compressible Navier-Stokes equations

$$
\begin{gathered}
\partial_{t} \rho+\nabla \cdot(\rho \boldsymbol{u})=0, \\
\rho\left(\partial_{t} \boldsymbol{u}+\boldsymbol{u} \cdot \nabla \boldsymbol{u}\right)=-\nabla \cdot \boldsymbol{P},
\end{gathered}
$$

where

$$
\begin{gathered}
\rho=\sum_{i} f_{i}, \\
\boldsymbol{u}=\sum_{i} \xi_{i} f_{i}, \\
\boldsymbol{P}=\sum_{i}\left(\boldsymbol{\xi}_{i}-\boldsymbol{u}\right)\left(\boldsymbol{\xi}_{i}-\boldsymbol{u}\right) f_{i}=\boldsymbol{P}^{(0)}+\boldsymbol{P}^{(1)}=\boldsymbol{I} p-2 \rho \nu \boldsymbol{S},
\end{gathered}
$$

where $\boldsymbol{S}=\left(\boldsymbol{\nabla} \boldsymbol{u}+(\boldsymbol{\nabla u})^{\mathrm{T}}\right) / 2$ is the strain-rate tensor, $p=c_{s}^{2} \rho$ is the pressure (this is the perfect gas law) and $v=c_{s}^{2} \tau$ is the kinematic viscosity. Another very interesting property 
of the Chapman-Enskog expansion of Equation (2) is that the off-equilibrium coefficients are also related between themselves by a recursive relation (as are the equilibrium ones) and are given by

$$
a_{1, \alpha_{1} \cdots \alpha_{n}}^{(n)}=a_{1, \alpha_{1} \cdots \alpha_{n-1}}^{(n-1)} u_{\alpha_{n}}+\left(u_{\alpha_{1}} \cdots u_{\alpha_{n-2}} a_{1, \alpha_{n-1} \alpha_{n}}^{(2)}+\operatorname{perm}\left(\alpha_{n}\right)\right),
$$

where 'perm $\left(\alpha_{n}\right)$ ' stands for all the cyclic index permutations of indexes from $\alpha_{1}$ to $\alpha_{n-1}$ ( $\alpha_{n}$ is never permuted), and where

$$
a_{1, \alpha \beta}^{(2)}=-2 \rho \tau c_{s}^{2} S_{\alpha \beta},
$$

with $S_{\alpha \beta}=\left(\partial_{\alpha} u_{\beta}+\partial_{\beta} u_{\alpha}\right) / 2$ is the strain rate tensor. Until this point we made the hidden assumption that the discrete Hermite polynomials $\mathcal{H}_{i}^{(n)}$ have the same orthogonality relations between themselves that the continuous Hermite polynomials up to an arbitrary order $n$. In reality only, a limited number of Hermite polynomials possess this property: for the standard lattices D3Q15, D3Q19 and D3Q27 this is true up to order $n=2$ (see [41] for example). In [37], one uses the D3Q27 lattice because of its better isotropy and therefore higher resemblance with the continuous case at the expense of a higher computational cost. To lower the amount of computations needed per grid point, we use here the D3Q19 quadrature.

The Hermite polynomials used here are a bit different than for the D3Q27 case. Here we will use only combinations of third-order Hermite polynomials which have the correct orthogonality properties. These are

$$
\begin{aligned}
& \mathcal{H}_{\alpha, x x y}^{(3)}+\mathcal{H}_{\alpha, y z z}^{(3)}, \\
& \mathcal{H}_{\alpha, x z z}^{(3)}+\mathcal{H}_{\alpha, x y y}^{(3)}, \\
& \mathcal{H}_{\alpha, y y z}^{(3)}+\mathcal{H}_{\alpha, x x z}^{(3)}, \\
& \mathcal{H}_{\alpha, x x y}^{(3)}-\mathcal{H}_{\alpha, y z z}^{(3)}, \\
& \mathcal{H}_{\alpha, x z z}^{(3)}-\mathcal{H}_{\alpha, x y y}^{(3)}, \\
& \mathcal{H}_{\alpha, y y z}^{(3)}-\mathcal{H}_{\alpha, x x z}^{(3)} .
\end{aligned}
$$

These Hermite polynomials being added to the set of $\mathcal{H}^{(0)}, \mathcal{H}^{(1)}$ and $\mathcal{H}^{(2)}$ the equilibrium distribution function is given by

$$
\begin{aligned}
f_{i}^{(0)}= & w_{i}\left(\rho+\frac{\boldsymbol{\xi}_{i} \cdot(\rho \boldsymbol{u})}{c_{s}^{2}}+\frac{1}{2 c_{s}^{4}} \mathcal{H}_{i}^{(2)}: \boldsymbol{a}_{0}^{(2)}+\frac{1}{2 c_{s}^{6}}\left(\mathcal{H}_{i, x x y}^{(3)}+\mathcal{H}_{i, y z z}^{(3)}\right)\left(a_{0, x x y}^{(3)}+a_{0, y z z}^{(3)}\right)\right. \\
& +\frac{1}{2 c_{s}^{6}}\left(\mathcal{H}_{i, x z z}^{(3)}+\mathcal{H}_{i, x y y}^{(3)}\right)\left(a_{0, x z z}^{(3)}+a_{0, x y y}^{(3)}\right) \\
& +\frac{1}{2 c_{s}^{6}}\left(\mathcal{H}_{i, y y z}^{(3)}+\mathcal{H}_{i, x x z}^{(3)}\right)\left(a_{0, y y z}^{(3)}+a_{0, x x z}^{(3)}\right)
\end{aligned}
$$




$$
\begin{aligned}
& +\frac{1}{6 c_{s}^{6}}\left(\mathcal{H}_{i, x x y}^{(3)}-\mathcal{H}_{i, y z z}^{(3)}\right)\left(a_{0, x x y}^{(3)}-a_{0, y z z}^{(3)}\right) \\
& +\frac{1}{6 c_{s}^{6}}\left(\mathcal{H}_{i, x z z}^{(3)}-\mathcal{H}_{i, x y y}^{(3)}\right)\left(a_{0, x z z}^{(3)}-a_{0, x y y}^{(3)}\right) \\
& \left.+\frac{1}{6 c_{s}^{6}}\left(\mathcal{H}_{i, y y z}^{(3)}-\mathcal{H}_{i, x x z}^{(3)}\right)\left(a_{0, y y z}^{(3)}-a_{0, x x z}^{(3)}\right)\right)
\end{aligned}
$$

and the off-equilibrium is

$$
\begin{aligned}
f_{i}^{(1)}= & w_{i}\left(\frac{1}{2 c_{s}^{4}} \mathcal{H}_{i}^{(2)}: \boldsymbol{a}_{1}^{(2)}+\frac{1}{2 c_{s}^{6}}\left(\mathcal{H}_{i, x x y}^{(3)}+\mathcal{H}_{i, y z z}^{(3)}\right)\left(a_{1, x x y}^{(3)}+a_{1, y z z}^{(3)}\right)\right. \\
& +\frac{1}{2 c_{s}^{6}}\left(\mathcal{H}_{i, x z z}^{(3)}+\mathcal{H}_{i, x y y}^{(3)}\right)\left(a_{1, x z z}^{(3)}+a_{1, x y y}^{(3)}\right) \\
& +\frac{1}{2 c_{s}^{6}}\left(\mathcal{H}_{i, y y z}^{(3)}+\mathcal{H}_{i, x x z}^{(3)}\right)\left(a_{1, y y z}^{(3)}+a_{1, x x z}^{(3)}\right) \\
& +\frac{1}{6 c_{s}^{6}}\left(\mathcal{H}_{i, x x y}^{(3)}-\mathcal{H}_{i, y z z}^{(3)}\right)\left(a_{1, x x y}^{(3)}-a_{1, y z z}^{(3)}\right) \\
& +\frac{1}{6 c_{s}^{6}}\left(\mathcal{H}_{i, x z z}^{(3)}-\mathcal{H}_{i, x y y}^{(3)}\right)\left(a_{1, x z z}^{(3)}-a_{1, x y y}^{(3)}\right) \\
& \left.+\frac{1}{6 c_{s}^{6}}\left(\mathcal{H}_{i, y y z}^{(3)}-\mathcal{H}_{i, x x z}^{(3)}\right)\left(a_{1, y y z}^{(3)}-a_{1, x x z}^{(3)}\right)\right)
\end{aligned}
$$

where

$$
\begin{gathered}
a_{1, \alpha \beta}^{(2)}=\sum_{i} \mathcal{H}_{i, \alpha \beta}^{(2)}\left(f_{i}-f_{i}^{(0)}\right)=-2 \rho \tau c_{s}^{2} S_{\alpha \beta}, \\
a_{1, \alpha \beta \gamma}^{(3)}=u_{\alpha} a_{1, \beta \gamma}^{(2)}+u_{\beta} a_{1, \gamma \alpha}^{(2)}+u_{\gamma} a_{1, \alpha \beta}^{(2)} .
\end{gathered}
$$

With these two modifications to the regularised model (see Equation 4), we have now the recursive regularised BGK Lattice-Boltzmann method (RR-BGK).

\section{A new improved RR-BGK collision operator}

In this paper, we propose a modified version of the regularisation procedure, which relies on the hybridisation of the computation of the velocity gradients with finite difference schemes in the computation of the recursive reconstruction parameters discussed in the preceding section. The deviatoric stress tensor in the above equation is replaced by a stabilising hybrid stress tensor $\tilde{\boldsymbol{P}}^{(1)}$ which reads

$$
\tilde{\boldsymbol{P}}^{(1)}=\boldsymbol{P}^{(1)} \sigma-(1-\sigma) 2 \rho \tau c_{s}^{2} \boldsymbol{S}^{F D}, \quad 0 \leq \sigma \leq 1
$$

where $\sigma=1$ is equivalent to the RR-BGK model. 
Using the simple second-order centred finite difference approximation

$$
\frac{g(x+\Delta x)-g(x-\Delta x)}{2 \Delta x}=\partial_{x} g(x)+\frac{1}{6} \Delta x^{2} \partial_{x}^{3} g(x),
$$

the finite-difference strain rate tensor $S^{F D}$ can be evaluated as

$$
\begin{aligned}
S_{\alpha \beta}^{F D} & \cong \frac{1}{2}\left(\partial_{\alpha} u_{\beta}+\frac{1}{6} \Delta x^{2} \partial_{\alpha}^{3} u_{\beta}+\partial_{\beta} u_{\alpha}+\frac{1}{6} \Delta x^{2} \partial_{\beta}^{3} u_{\alpha}\right), \\
& \cong \frac{1}{2}\left(\partial_{\alpha} u_{\beta}+\partial_{\beta} u_{\alpha}+\frac{1}{6} \Delta x^{2}\left(\partial_{\alpha}^{3} u_{\beta}+\partial_{\beta}^{3} u_{\alpha}\right)\right), \\
& \cong S_{\alpha \beta}+\frac{1}{2}\left(\frac{1}{6} \Delta x^{2}\left(\partial_{\alpha}^{3} u_{\beta}+\partial_{\beta}^{3} u_{\alpha}\right)\right),
\end{aligned}
$$

where $S_{\alpha \beta}$ denotes the exact velocity gradient tensor.

The collision operator then reads

$$
\Omega=-\frac{1}{\tau} \tilde{f}_{i}^{(1)},
$$

where

$$
\tilde{f}_{i}^{(1)}=f_{i}^{(1)} \sigma-(1-\sigma) \frac{\rho \tau}{c_{s}^{2}} \mathcal{H}_{i}^{(2)}: S^{F D},
$$

where $f_{i}^{(1)}$ is given by Equation (28). A more efficient way to compute this last term is to evaluate it through the recursive relation using a following modified expression for the coefficients:

$$
\tilde{f}_{i}^{(1)}=f_{i}^{(1)}\left(\tilde{a}_{1, \alpha \beta}^{(2)}\right) \text {, }
$$

with $f_{i}^{(1)}$ still given in Equation (28) and where $\tilde{a}_{1, \alpha \beta}^{(2)}$ is

$\tilde{a}_{1, \alpha \beta}^{(2)}=\sigma \sum_{i} \mathcal{H}_{i, \alpha \beta}^{(2)}\left(f_{i}-f_{i}^{(0)}\right)+(1-\sigma)\left(-2 \rho \tau c_{s}^{2} S_{\alpha \beta}\right)=\sigma a_{1, \alpha \beta}^{(2)}+(1-\sigma)\left(-2 \rho \tau c_{s}^{2} S_{\alpha \beta}\right)$.

The actual Boltzmann equation we will solve hereafter is given by

$$
\partial_{t} f_{i}+\boldsymbol{\xi}_{i} \cdot \nabla f_{i}=-\frac{1}{\tau}\left(f_{i}^{(1)} \sigma-(1-\sigma) \frac{\rho \tau}{c_{s}^{2}} \mathcal{H}_{i}^{(2)}: S^{F D}\right) .
$$

Performing the Chapman-Enskog expansion on this equation, one gets at the lowest order

$$
\partial_{t} f_{i}^{(0)}+\boldsymbol{\xi}_{i} \cdot \nabla f_{i}^{(0)}=-\frac{1}{\tau}\left(f_{i}^{(1)} \sigma-(1-\sigma) \frac{\rho \tau}{c_{s}^{2}} \mathcal{H}_{i}^{(2)}: S^{F D}\right) .
$$

Taking the zeroth-, first- and second-order moments of this last equation, one gets

$$
\begin{gathered}
\partial_{t} \rho+\nabla \cdot(\rho \boldsymbol{u})=0, \\
\partial_{t}(\rho \boldsymbol{u})+\nabla \cdot(\rho \boldsymbol{u} \boldsymbol{u})=-\nabla p,
\end{gathered}
$$




$$
\partial_{t}(\rho \boldsymbol{u} \boldsymbol{u})+\nabla \cdot(\rho \boldsymbol{u} \boldsymbol{u} \boldsymbol{u})+\nabla(\rho \boldsymbol{u})+(\nabla(\rho \boldsymbol{u}))^{\mathrm{T}}=-\frac{1}{\tau} \tilde{\boldsymbol{P}}^{(1)} .
$$

Using the first two equations, the third can be rewritten as

$$
-2 \rho c_{s}^{2} \tau \boldsymbol{S}+\mathcal{O}\left(\mathrm{Ma}^{3}\right)=\tilde{\boldsymbol{P}}^{(1)},
$$

where $\mathrm{Ma}=u / c_{s} \ll 1$ is the Mach number. The Mach number is shown to scale like Ma $\Delta t / \Delta x$ (see [35] among others). Therefore using the diffusive limit for the scaling of $\Delta t$, $\Delta t \sim \Delta x^{2}$, which is the limit consistent with incompressible fluids, one can safely neglect the $\mathcal{O}\left(\mathrm{Ma}^{3}\right)$ term which scales like $\Delta x^{3}$.

Therefore the previous equation can be rewritten as

$$
\begin{aligned}
\sigma P_{\alpha \beta}^{(1)} & =-2 \rho \tau c_{s}^{2} S_{\alpha \beta}+(1-\sigma) 2 \rho \tau c_{s}^{2} S_{\alpha \beta}^{F D}, \\
P_{\alpha \beta}^{(1)} & =-2 \rho \tau c_{s}^{2} S_{\alpha \beta}+\left(\frac{1-\sigma}{\sigma}\right) \rho \tau c_{s}^{2} \frac{\Delta x^{2}}{6}\left(\partial_{\alpha}^{3} u_{\beta}+\partial_{\beta}^{3} u_{\alpha}\right) .
\end{aligned}
$$

With this relation, one recovers the equivalent Navier-Stokes equation

$$
\begin{aligned}
\rho\left(\partial_{t} u_{\alpha}+u_{\beta} \partial_{\beta} u_{\alpha}\right)= & -\partial_{\alpha} p+2 \partial_{\beta}\left(\mu S_{\alpha \beta}\right) \\
& -\left(\frac{(1-\sigma) \Delta x^{2}}{6 \sigma}\right) \partial_{\beta}\left(\mu\left(\partial_{\alpha}^{3} u_{\beta}+\partial_{\beta}^{3} u_{\alpha}\right)\right),
\end{aligned}
$$

where $\mu=c_{s}^{2} \rho \tau$.

After performing the standard time-space discretisation using the trapezoidal approximation of Equation (38), one gets the following numerical scheme

$$
\bar{f}_{i}\left(\boldsymbol{x}+\boldsymbol{\xi}_{i}, t+1\right)=f_{i}^{e q}(\rho, \boldsymbol{u})+\left(1-\frac{1}{\bar{\tau}}\right) \tilde{f}_{i}^{(1)},
$$

where $\bar{f}_{i}=f_{i}+\frac{1}{2 \tau} \tilde{f}_{i}^{(1)}, \tilde{f}_{i}^{(1)}$ is given by Equation (36) and $\bar{\tau}=\tau+1 / 2$.

The present model is discretised in space using embedded subdomains with uniform mesh with a grid spacing ratio of two between two successive subdomains (see Figure 1). For all the grid points far from the transition region, the collision-propagation algorithm is applied and $\boldsymbol{S}^{F D}$ is computed using centred second-order finite differences schemes. As shown in Figure 1, the coarse grid $(\bigcirc)$ overlaps the fine grid $(\bullet)$ making it possible to apply the collision-propagation algorithm on the transition grid points belonging to the coarse grid $(\bigcirc)$. Because of a lack of neighbour points, the propagation step cannot be applied at the fine grid points $(\bullet)$ located in the transition layer. On these grid points, the macroscopic values $\left(\rho\right.$ and $\boldsymbol{u}$ ) and the deviatoric stress tensor $\left(\boldsymbol{P}^{(1)}\right)$ are interpolated from the coarse grid neighbours located in the transition layer and $S^{F D}$ is computed using non-centred first-order finite difference schemes.

\section{Associated kinetic energy dissipation, equivalent artificial viscosity and dynamic collision kernel}

The extra dissipation of kinetic energy associated to the use of the new hybrid collision kernel introduced above, denoted $\varepsilon_{\sigma}$, can be easily obtained considering the evolution 


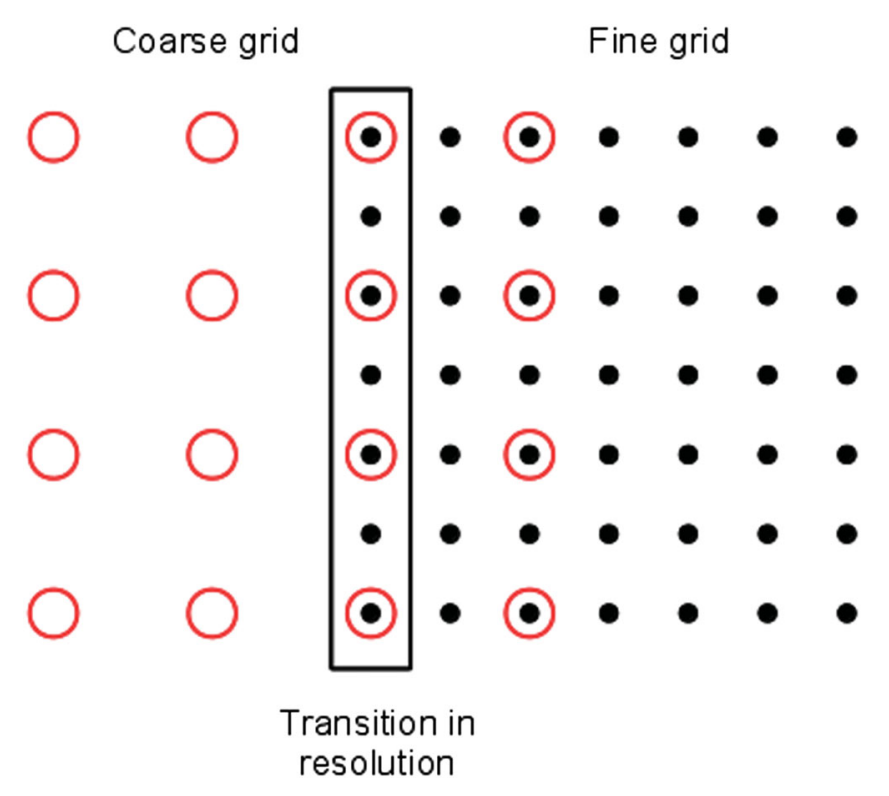

Figure 1. Visualisation of the grid arrangement around transition in resolution.

equation for the kinetic energy $\frac{1}{2} \boldsymbol{u} \cdot \boldsymbol{u}$ derived from Equation (45). After some algebra, one obtains

$$
\varepsilon_{\sigma}=v_{\sigma}\left|\nabla^{2} \boldsymbol{u}\right|^{2}, \quad v_{\sigma}=\frac{1-\sigma}{6 \sigma} \Delta x^{2} c_{s}^{2} \tau,
$$

where $v_{\sigma}$ is the $\sigma$-dependent artificial hyperviscosity associated to the hybrid RR-BGK collision operator. It is worth noting that the induced dissipation originates in the leading error term of the finite difference scheme used to compute the velocity derivatives. Therefore, the use of a second-order centred scheme leads to a bi-Laplacian-based hyperviscosity, i.e. a $v_{\sigma} \nabla^{4} \boldsymbol{u}$ correction in the momentum equation. Higher order artificial dissipation operators can be easily defined considering higher order centred finite difference schemes to compute $S_{i j}$. As a matter of fact, using a $p$ th-order scheme will introduce an hyperviscous operator proportional to $\nabla^{2 p} \boldsymbol{u}$. An increase in the order of the hyperviscosity is associated to an concentration of the induced dissipation at small resolved scales and to a possible way for smart control of spurious wiggles.

The above expression for the induced dissipation $\varepsilon_{\sigma}$ offers a smart way to obtain an ILES scheme by tuning $\sigma$ in order to recover the same dissipation as the one provided by a subgrid scale model, $\varepsilon_{\text {sgs }}$. Considering a generic subgrid viscosity $v_{t}$ (the same development holds for any RANS eddy viscosity model), one has $\varepsilon_{\text {sgs }}=v_{t}|\nabla \boldsymbol{u}|^{2}$. Therefore, equalising the artificial and the subgrid dissipation leads to

$$
v_{t}|\nabla \boldsymbol{u}|^{2}=v_{\sigma}\left|\nabla^{2} \boldsymbol{u}\right|^{2},
$$

whose solution is

$$
v_{\sigma}=L_{V K}^{2} v_{t}
$$


where $L_{V K}=|\nabla \boldsymbol{u}| /\left|\nabla^{2} \boldsymbol{u}\right|$ can be interpreted as a renormalised extended definition of the von Kármán length scale [42]. The associated value of $\sigma$ is

$$
\sigma=\frac{1}{6 v_{t} \frac{L_{V K}^{2}}{\Delta x^{2} c_{s}^{2} \tau}+1}
$$

These expressions can be further simplified considering subgrid viscosity models that can be written as $\nu_{t}=c_{s g s} \Delta x^{2} / \tau_{s g s}$ where $\tau_{s g s}$ and $c_{s g s}$ are the subgrid time scale and the subgrid model constant, respectively. The expression for the tuning parameter is

$$
\sigma=\frac{1}{6 \frac{c_{s g s} L_{V K}^{2}}{c_{s}^{2} \tau \tau_{s g s}}+1} .
$$

It is worth noting that this last expression does not explicitly involve the mesh size $\Delta x$, leading to an easy implementation in multiresolution algorithms.

The last relation can be inverted to find the value of the Smagorinsky constant that yields the same dissipation as the regularised collision kernel:

$$
c_{s g s}=\left(\frac{1}{\sigma}-1\right) \frac{c_{s}^{2} \tau \tau_{s g s}}{6 L_{V K}^{2}} .
$$

In the case, the regularised collision kernel is used in the ILES mode, the influence of the implicit subgrid dissipation can be evaluated thanks to the subgrid viscosity parameter $s$ defined as [43]

$$
s=\frac{\varepsilon_{\sigma}}{\varepsilon_{\sigma}+\varepsilon_{v}}
$$

where $\varepsilon_{v}=v|\nabla \boldsymbol{u}|^{2}$ denotes the molecular viscosity-induced dissipation. After some algebra, one obtains

$$
\frac{1}{s}=1+\frac{\varepsilon_{v}}{\varepsilon_{\sigma}}=1+\frac{v}{v_{\sigma}} L_{V K}^{2}=1+\frac{6 \sigma \nu}{(1-\sigma) \Delta x^{2} c_{s}^{2} \tau} L_{V K}^{2} .
$$

\section{Vreman's explicit subgrid model}

The explicit subgrid model selected in the present paper for both explicit LES and ILES simulations is the one proposed by Vreman [44], which is observed to have very interesting self-adaptation features without relying on a test filter-based procedure or a prognostic equation for a subgrid quantity. More specifically, this model is observed to behave in a very satisfactory manner in fully developed turbulent shear flows, and also in transitional flows and near wall region without the use of dynamic procedure, test filter and semiempirical stabilisation step such as averaging or clipping. It is also computationally very efficient, since it does not involve the computation of eigenvalues. A complete analysis of its properties, including symmetry preservation, is available in $[45,46]$. 
The subgrid tensor is defined as

$$
R_{i j}=\overline{u_{i} u_{j}}-\overline{u_{i}} \overline{u_{j}},
$$

where the bar symbol denotes the LES filter. It is modelled as

$$
R_{i j} \simeq-2 v_{t} S_{i j}+R_{k k} \delta_{i j}
$$

where viscosity is defined as

$$
v_{t}=c \sqrt{\frac{B_{\beta}}{\alpha_{i j} \alpha_{i j}}},
$$

with

$$
\alpha_{i j}=\frac{\partial \bar{u}_{i}}{\partial x_{j}}, \quad \beta_{i j}=\Delta^{2} \alpha_{i j} \alpha_{i j}
$$

and

$$
B_{\beta}=\beta_{11} \beta_{22}-\beta_{12}^{2}+\beta_{11} \beta_{33}-\beta_{13}^{2}+\beta_{22} \beta_{33}-\beta_{23}^{2} .
$$

The cutoff length $\Delta$ is taken equal to the mesh size $\Delta_{x}$ in the present LBM-based simulations, which are carried out on grids with cubic cells. The model constant is taken equal to $c=2.5 C_{S}^{2}$, where $C_{S}=0.18$ is the Smagorinsky constant.

In the classical LES mode, this model is implemented by modifying the relaxation time $\tau$ in the BGK collision kernel according to

$$
\tau=\frac{v+v_{t}}{c_{s}^{2}}+\frac{\Delta t}{2}
$$

where $v$ denotes the fluid molecular viscosity.

\section{Application to the flow around a cylinder at $\operatorname{Re}=3900$}

\subsection{Case description}

The new Hybrid RR-BGK collision operator is assessed considering the flow around a cylinder at $\operatorname{Re}=U_{0} D / v=3900$ and $M a=U_{0} / C_{0}=0.0585$ with $U_{0}$ the inflow velocity, $C_{0}$ the speed of sound and $D$ the cylinder diameter. Four different computational cases are defined, in order to assess both LES and ILES capabilities of the method (see Table 1). Case 1 and Case 2 correspond to ILES simulations with constant values of the weighting parameter $\sigma$. Case 3 is an explicit LES simulation, in which $\sigma=0.99$, i.e. the total amount of numerical dissipation is expected to remain very small. The last test case (Case 4) corresponds to an ILES simulation with a dynamic evaluation of the parameter $\sigma$, Vreman's

Table 1. Computation parameters.

\begin{tabular}{lcc}
\hline Case & Subgrid model & $\sigma$ \\
\hline Case 1 & - & 0.97 \\
Case 2 & - & 0.985 \\
Case 3 & Vreman & 0.99 \\
Case 4 & Vreman & Dynamic \\
\hline
\end{tabular}




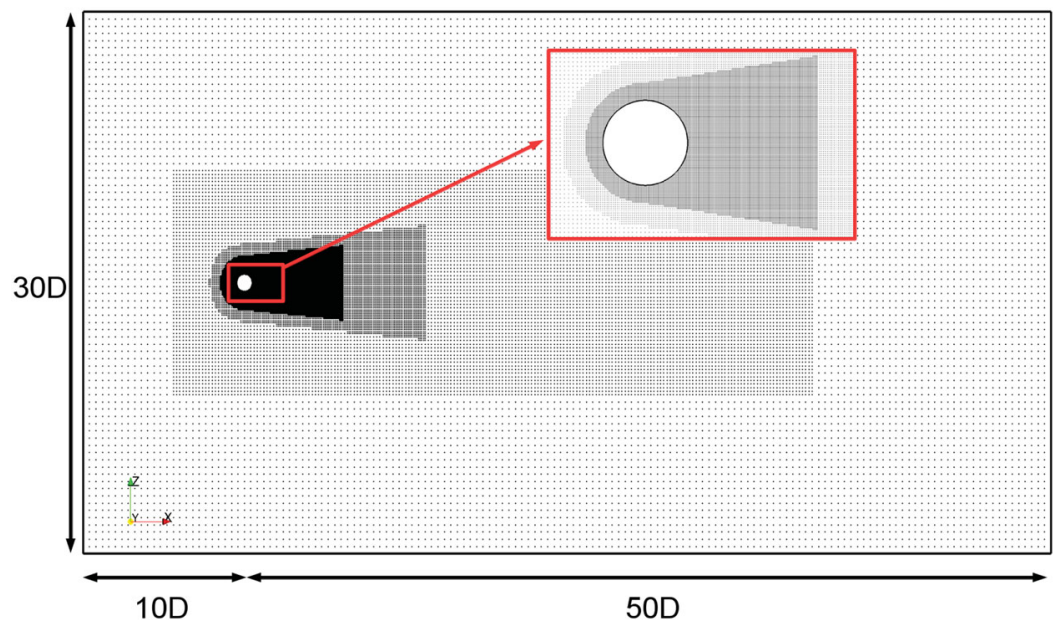

Figure 2. View of the computational domain and the grid points.

subgrid model being used to evaluate the targeted dissipation. The computation of $\sigma$ in that case follows the procedure described in Section 3 for $\rho$ and $\boldsymbol{u}$ for the transition in resolution fine grid points.

The computational domain (see Figure 2) is extended 9.5 diameter upstream the cylinder and 49.5 diameter downstream the cylinder. A non-reflective subsonic outflow [47] is used for the outlet and free slip conditions are imposed in the top and bottom boundaries 14.5 diameter far from the cylinder leading to a blockage ratio of 3.3\%. The spanwise extent of the computational domain is taken equal to $4 D$ and is used with periodic boundaries. This spanwise size is known to prevent spurious effects due to unphysical correlations that may be induced by periodic boundary conditions. It is shown in Breuer [48] that no differences are obtained on average quantities using $\pi D$ or $2 \pi D$ for the spanwise size.

The computational grid (see Figure 2) is composed of several embedded volumes of uniform cartesian mesh. The grid size is reduced from $D / 2.5$ far from the cylinder to $D / 80$ (finer than in Parnaudeau et al. [49]) around the cylinder and in its wake. A layer of 15 grid points with $\Delta x=D / 80$ is applied around the cylinder and extended 1.5 diameter downstream it $(x=2 D)$ to ensure a good representation of the recirculation bubble. The refinement areas with $\Delta x=D / 40$ and $\Delta x=D / 20$ are applied on 10 grid point layers around the cylinder and extended 3.5 and 5.5 diameter downstream the cylinder $(x=4 D$ and $x=6 D$ ). The distance of the first node to the cylinder is between 0 and $0.0177 D$ with an averaged value of $0.00865 \mathrm{D}$ which is lower than in Alkishriwi et al. [50] and Ouvrard et al. [51]. The use of cubic cells leads to 320 grid points in the spanwise direction for the minimal grid size area and a total of 10 million grid points were used in this computation. The computational time was around $47 \mathrm{CPU}$ hours per vortex shedding period on 48 processors. Statistically steady state was reached after $93 T^{*}$ with $T^{*}=D / U_{0}$ and all the statistics presented in Section 6.2 were computed on $84 T^{*}(\approx 17$ vortex shedding periods).

\subsection{Results}

Key parameters related to the mean flow are reported in Table 2 with reference experimental and numerical results. It is observed that the mean drag coefficient $C_{d}$ and 
Table 2. Overview of numerical and experiment results.

\begin{tabular}{|c|c|c|c|c|c|c|}
\hline Case & Model & $C_{d}$ & $C_{1}^{\prime}$ & St & $L_{r} / D$ & $-C p_{b}$ \\
\hline $\begin{array}{l}\text { Parnaudeau et al. } \\
\text { [49] }\end{array}$ & PIV & & & 0.208 & 1.51 & \\
\hline Dong et al. [52] & DNS & & & $0.206-0.210$ & $1-1.18$ & $0.93-1.04$ \\
\hline Ma et al. [53] & DNS & $0.84-1.04$ & & $0.203-0.219$ & $1-1.59$ & \\
\hline Alemi et al. [54] & LES-Smag & $0.92-1.01$ & $0.07-0.14$ & $0.205-0.225$ & & \\
\hline Alkishriwi et al. [50] & LES & 1.05 & & 0.217 & 1.31 & \\
\hline \multirow[t]{2}{*}{ Breuer [48] } & LES-Smag & $0.969-1.486$ & & & $0.397-1.686$ & $0.687-1.665$ \\
\hline & LES-DynSmag & $1.016-1.071$ & & & $1.197-1.372$ & $0.941-1.011$ \\
\hline Mani et al. [55] & LES & 0.99 & & 0.206 & & 0.86 \\
\hline $\begin{array}{l}\text { Franke and Frank } \\
\text { [56] }\end{array}$ & LES & $0.978-1.005$ & & 0.209 & $1.34-1.64$ & $0.85-0.94$ \\
\hline $\begin{array}{l}\text { Kravchenko and } \\
\text { Moin [57] }\end{array}$ & LES & 1.04 & & 0.210 & 1.35 & 0.94 \\
\hline \multirow[t]{2}{*}{ Lysenko et al. [58] } & LES-TKE & 0.97 & 0.09 & 0.209 & 1.67 & 0.91 \\
\hline & LES-Smag & 1.18 & 0.44 & 0.19 & 0.9 & 0.8 \\
\hline Meyer et al. [59] & LES & $1.05-1.07$ & & $0.21-0.215$ & $1.18-1.38$ & $0.92-1.05$ \\
\hline \multirow[t]{4}{*}{ Ouvrard et al. [51] } & LES-Smag & 0.99 & 0.125 & 0.218 & 1.54 & 0.85 \\
\hline & LES-Vreman & 0.92 & 0.054 & 0.227 & 1.83 & 0.78 \\
\hline & LES-WALE & 1.02 & 0.219 & 0.221 & 1.22 & 0.94 \\
\hline & ILES & 0.92 & 0.052 & 0.225 & 1.85 & 0.77 \\
\hline $\begin{array}{l}\text { Parnaudeau et al. } \\
\text { [49] }\end{array}$ & LES & & & 0.208 & 1.56 & \\
\hline $\begin{array}{l}\text { Abrahamsen Prsic } \\
\text { et al. [60] }\end{array}$ & LES & $1.0784-1.2365$ & $0.1954-0.4490$ & $0.1956-0.2152$ & 1.27 & \\
\hline Wormon et al. [61] & LES-WALE & 0.99 & 0.108 & 0.21 & 1.45 & 0.88 \\
\hline Zhang et al. [62] & LES & $1.001-1.098$ & $0.125-0.345$ & $0.21-0.22$ & & \\
\hline \multirow{3}{*}{$\begin{array}{l}\text { D'Alessandro et al. } \\
\text { [63] }\end{array}$} & SA DES & $1.205-1.2776$ & $0.428-0.6140$ & $0.204-0.215$ & $0.7172-0.85$ & $1.077-1.289$ \\
\hline & SA IDDES & $1.0235-1.4106$ & $0.1458-0.8283$ & $0.205-0.222$ & $0.5137-1.4270$ & $0.8780-1.4688$ \\
\hline & $v^{2}-f \mathrm{DES}$ & $0.9857-1.2553$ & $0.1088-0.5719$ & $0.205-0.214$ & $0.7270-1.6780$ & $0.8290-1.2570$ \\
\hline Case 1 & ILES & 0.936 & 0.044 & 0.212 & 2.05 & 0.779 \\
\hline Case 2 & ILES & 0.973 & 0.077 & 0.210 & 1.835 & 0.828 \\
\hline Case 3 & LES-Vreman & 0.954 & 0.048 & 0.209 & 2.04 & 0.779 \\
\hline Case 4 & ILES & 1.047 & 0.165 & 0.212 & 1.425 & 0.925 \\
\hline
\end{tabular}

Note: Smag stands for Smagorinsky model, DynSmag for Dynamic Smagorinsky model, TKE for Turbulent Kinetic Energy model, SA for Spalart Allmaras and IDDES for Improved Delayed DES.

the Strouhal number St associated to the main frequency of aerodynamic forces exerted on the cylinder are very accurately recovered in all cases. The predicted values of the rms value of the fluctuations of the lift coefficient $C_{l}^{\prime}$, the base pressure coefficient $C p_{b}$ and the normalised recirculation bubble length $L_{r} / D$ exhibit more dispersion, but always remain within the range of variation of previous DNS (Direct Numerical Simulation), LES and DES (Detached Eddy Simulation) results. But it is worth noting that a too large value of $L_{r}$ is found in cases 1, 2 and 3 showing that the transition to turbulence in the separated shear layers present in the formation region is delayed when compared to Parnaudeau's experiments. On the overall, case 4 (ILES with dynamic tuning of the weighting parameter $\sigma$ ) yields a very good prediction of all parameters, followed by case 2 (ILES with a small constant value of $\sigma$ ).

A deeper insight into the results is obtained considering Figure 3, which displays the streamwise evolution of the mean longitudinal velocity along the symmetry axis in the cylinder wake. It is observed that in all cases the maximum amplitude of the reverse flow in the recirculation bubble is accurately predicted when compared to experimental data. It must also be noticed that the velocity in the far wake is also recovered in all cases, showing 


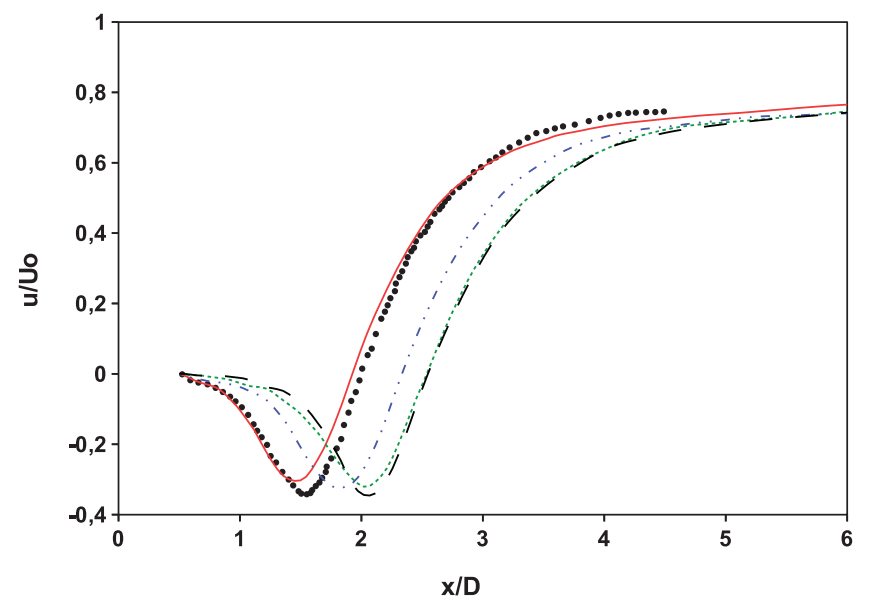

Figure 3. Mean streamwise velocity in the wake centreline: Parnaudeau et al. [49] PIV 1 - and PIV 2 *, present case $1 \ldots \ldots$, present case $2 \ldots \ldots \ldots$, present case $3 \ldots \ldots \ldots \ldots$, present case 4

that both domain size and boundary conditions are adequately chosen since there is no spurious mass/momentum leaks on upper and lower boundaries and that outflow boundary conditions do not induce unphysical mass/momentum flux. As mentioned above, case 4 leads to an almost perfect agreement with experimental results. Moreover it is observed in Figure 3 that the transition in resolution located at $x / D=2$ and $x / D=4$ does not include spurious effects on the velocity fields which demonstrates the good implementation of the present method.

The topology of the wake is now investigated looking at vertical profiles of the mean velocity at different location in the cylinder wake (see Figures 4 and 5). Once again, the simulation based on the dynamic version of the new HRR-BGK collision model (case 4) is in almost perfect agreement with experimental data. An important point is that a $\mathrm{V}$-shaped profile is recovered on the mean longitudinal velocity profile in agreement with experimental data, while many less accurate LES predict a U-shaped profile [49]. Discrepancies observed in the three other cases are mainly due to the error on the prediction of the length of $L_{r}$, but the global topology of both the mean recirculation bubble and near wake is recovered in all cases.

Since the size of the recirculation bubble is governed by the transition process in the separated shear layers, it is interesting to analyse the resolved Reynolds stresses. Vertical profiles of the longitudinal and vertical resolved Reynolds stresses at several locations in the wake are displayed in Figures 6 and 7. Once again, the very good accuracy of the case 4 is observed. In that case, the shear layer dynamics is very well recovered, since both the maximum value and the shear layer thickness and spreading rate are accurately predicted. In other cases, the separated shear layer spreading rate is underpredicted, leading to the prediction of a too long recirculation bubble.

This is further confirmed looking at the streamwise evolution of the resolved longitudinal Reynolds stress $\overline{u^{\prime} u^{\prime}}$ along the wake symmetry line that is displayed in Figure 8. Case 4 is in very good agreement with experimental data, since both the location and the amplitude of the peak located near the end of the recirculation bubble are satisfactorily predicted. In the three other cases, peaks are damped and shifted downstream, which is 

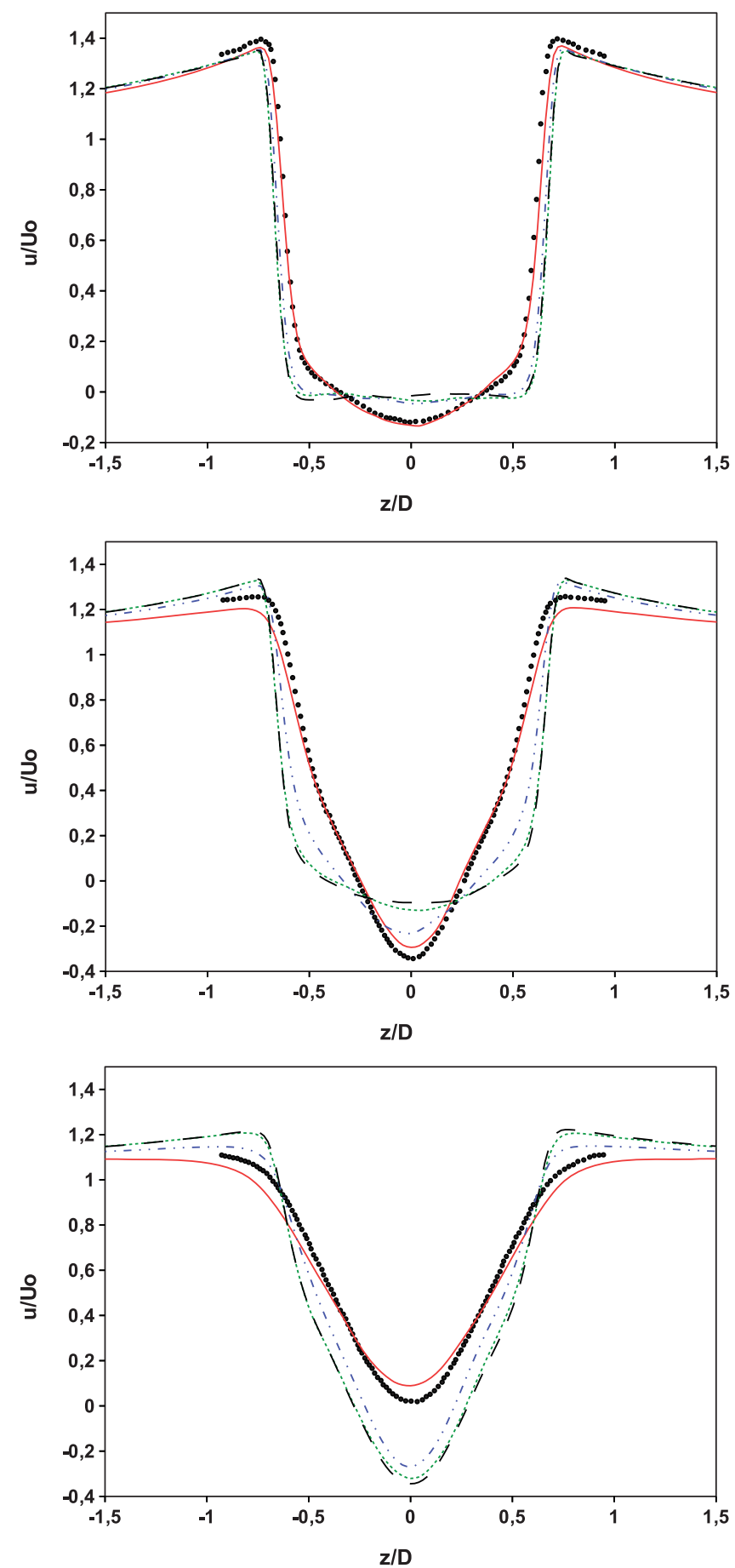

Figure 4. Mean streamwise velocity at $x=1.06 D$ (top), $x=1.54 D$ (middle) and $x=2.02 D$ (bottom) in the wake of the cylinder: Parnaudeau et al. [49] PIV 1 - and PIV $2{ }^{*}$, present case 1 - $\quad$, present case

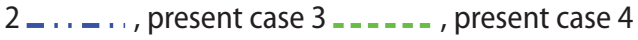



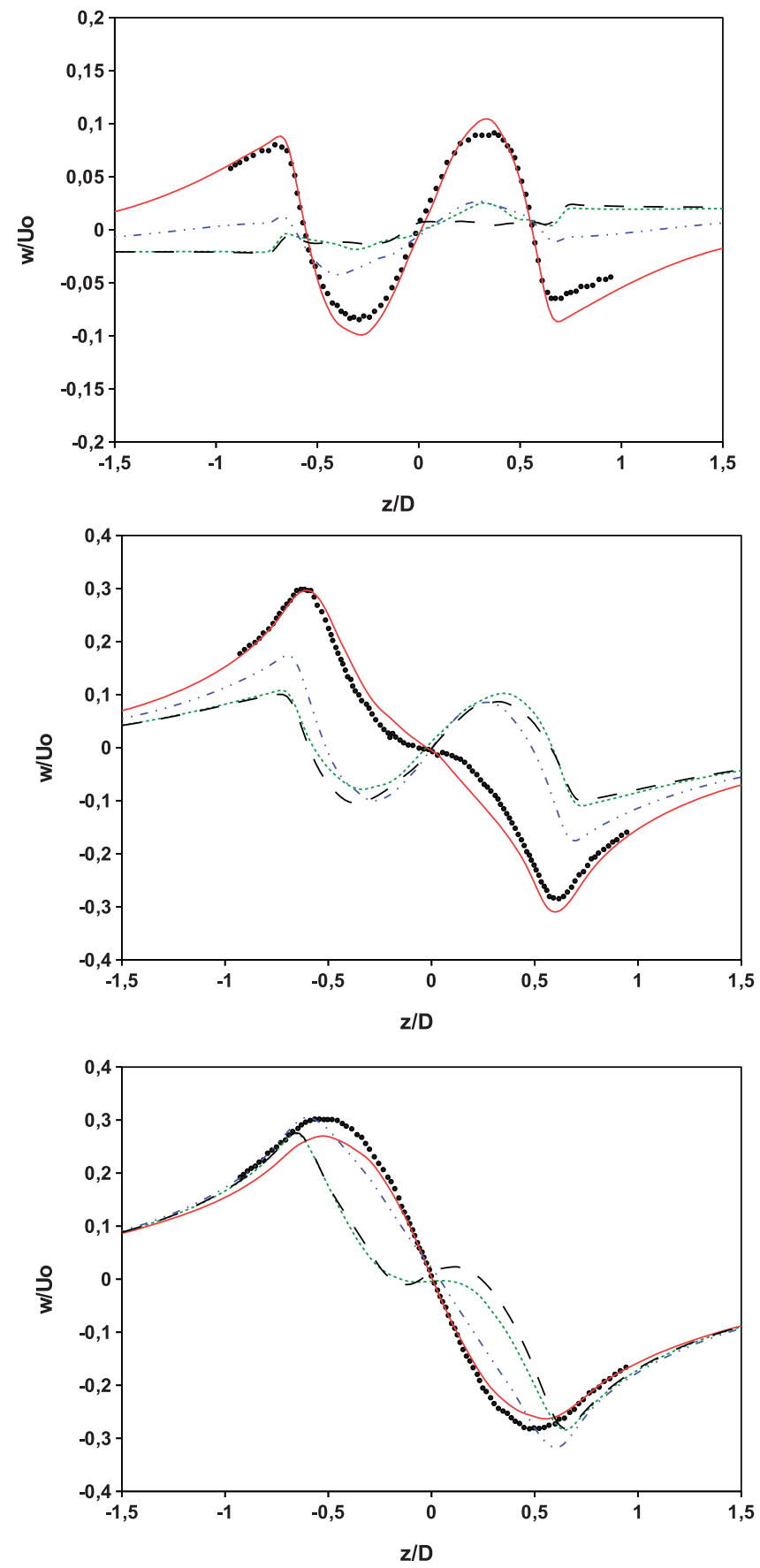

Figure 5. Mean normal velocity at $x=1.06 D$ (top), $x=1.54 D$ (middle) and $x=2.02 D$ (bottom) in the wake of the cylinder: Parnaudeau et al. [49] PIV $1 \cdot$ and PIV $2 *$, present case $1 \ldots$ $\ldots \ldots$...., present case 3 .....- , present case 4 

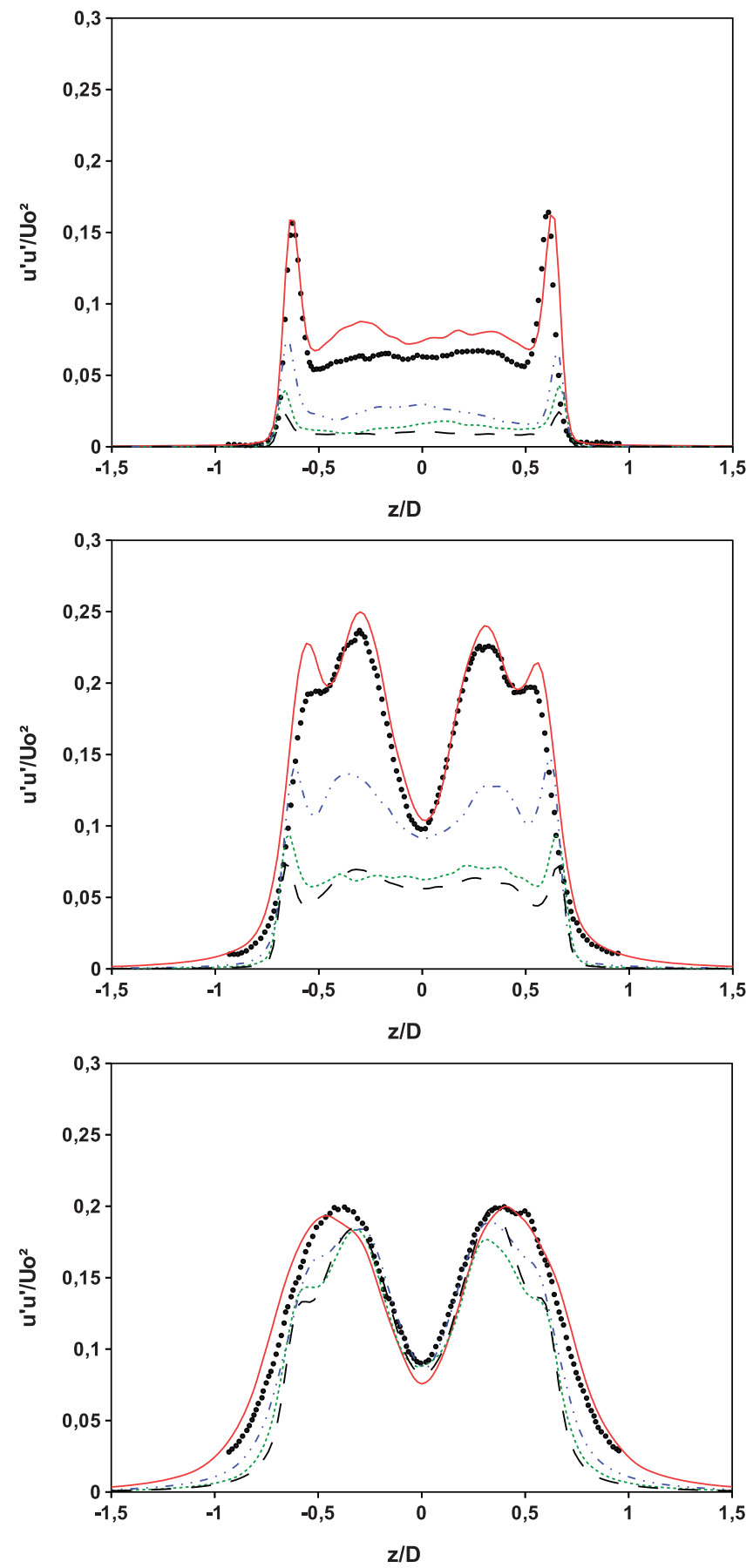

Figure 6. Vertical profiles of the streamwise resolved Reynolds stress $\overline{u^{\prime} u^{\prime}}$ at $x=1.06 D$ (top), $x=1.54 D$ (middle) and $x=2.02 D$ (bottom) in the wake of the cylinder: Parnaudeau et al. [49] PIV $1 \cdot$ and PIV 2 *, present case $1 \ldots \ldots$, present case $2 \ldots \ldots \ldots$, present case $3 \ldots \ldots \ldots$, present case 4 

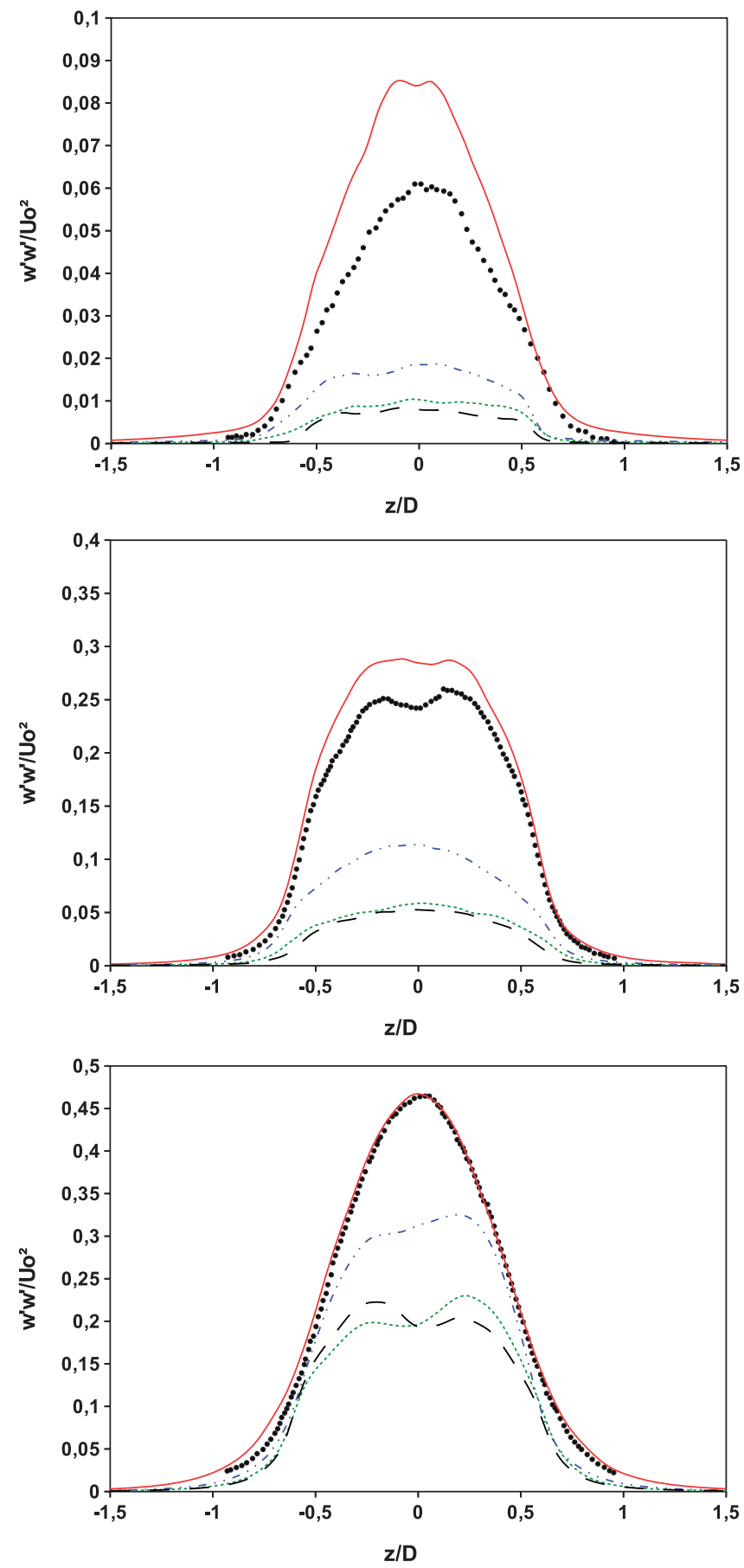

Figure 7. Vertical profiles of the normal resolved Reynolds stress $\overline{w^{\prime} w^{\prime}}$ at $x=1.06 D$ (top), $x=1.54 D$ (middle) and $x=2.02 D$ (bottom) in the wake of the cylinder: Parnaudeau et al. [49] PIV $1 \cdot$ and PIV 2 ${ }^{*}$, present case $1 \ldots \ldots$, present case $2_{\ldots} \ldots \ldots$. . , present case $3{ }_{-\ldots-\ldots-\ldots}$, present case 4 


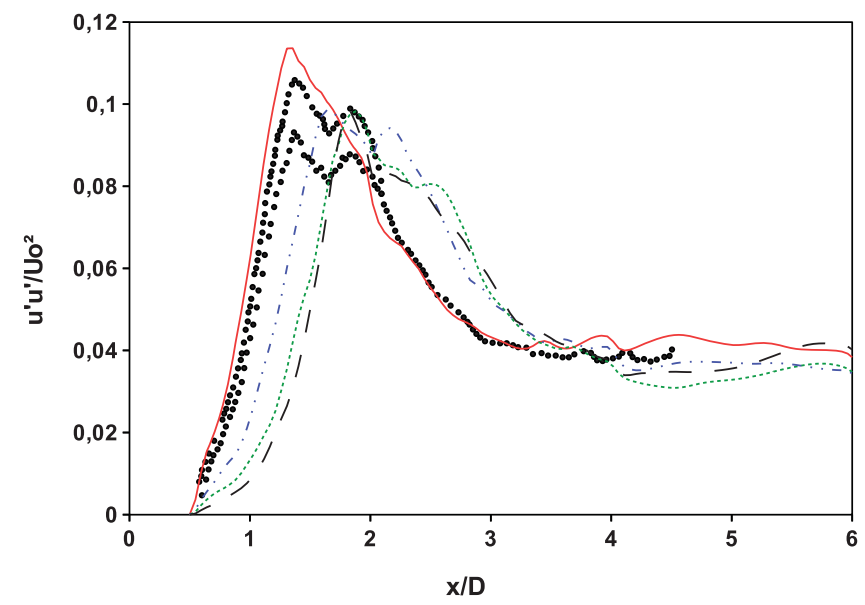

Figure 8. Variance of the streamwise velocity in the wake centreline: Parnaudeau et al. [49] PIV 1 and PIV $2{ }^{*}$, present case $1 \ldots \ldots$, present case $2 \ldots \ldots \ldots$, present case $3 \ldots \ldots \ldots$, present case 4
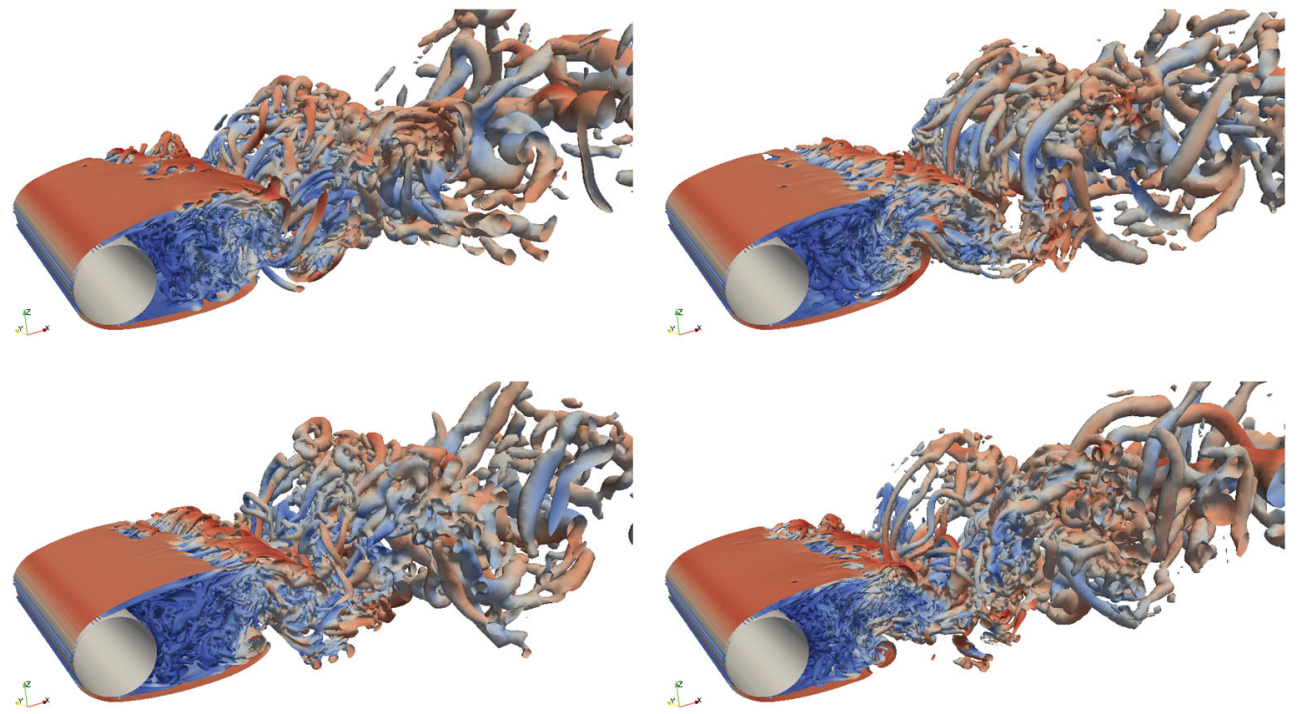

Figure 9. Iso contours of normalised $Q$ criterion $\left(Q^{*}=Q\left(D^{2} / U_{0}^{2}\right)=1\right)$ coloured by velocity magnitude: Case 1 (top left), Case 2 (top right), Case 3 (bottom left) and Case 4 (bottom right).

coherent with previous comments dealing with the damping of the shear layer dynamics in these simulations.

The effects of the dissipation on the flow structures are illustrated in Figures 9 and 10, which display iso-surfaces of instantaneous $\mathrm{Q}$ criterion. While it is seen that the flow physics is qualitatively well predicted in all cases (laminar boundary layers along the cylinder with laminar separation and transition in the separated shear layers, large-scale roll-up of the shear layers and appearance of small scale worm-like vortices in the wake), some subtle differences can be detected. As a matter of fact, it can be seen that small-scale structures 

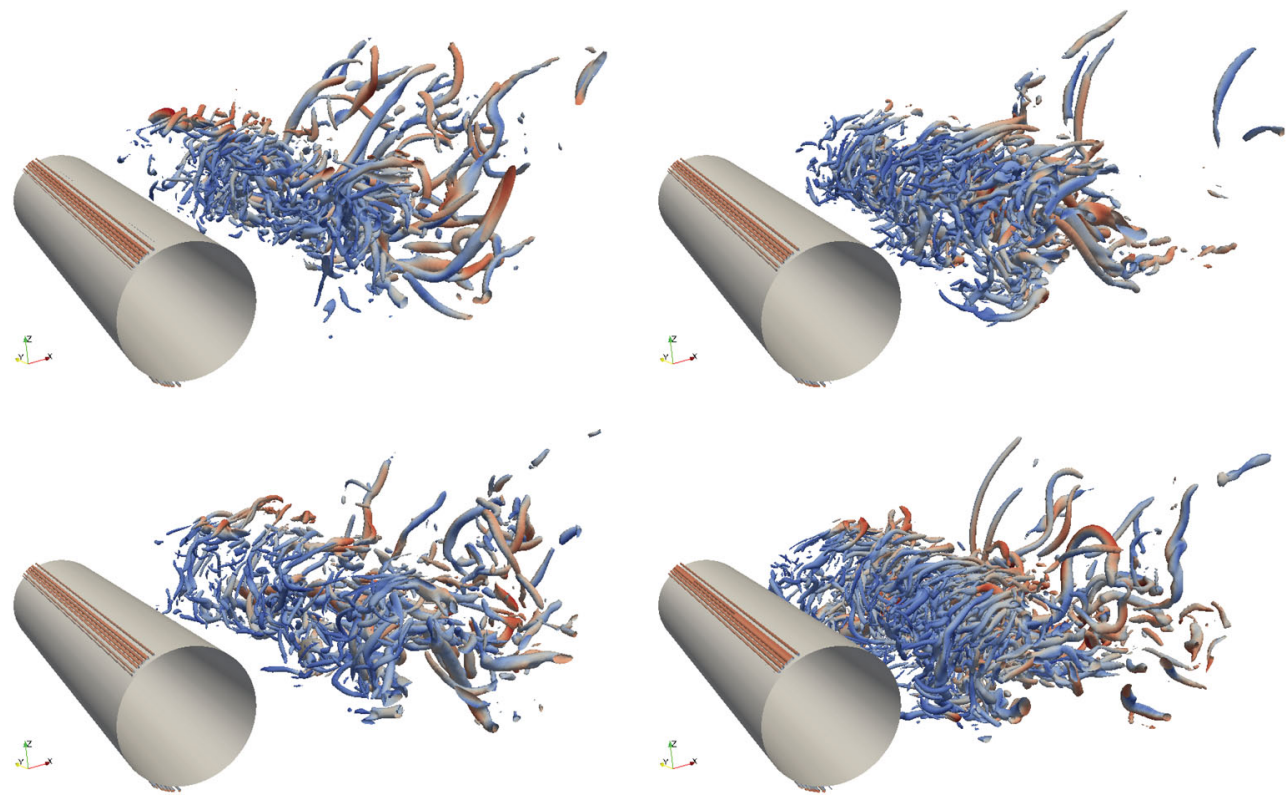

Figure 10. Iso contours of normalised $Q$ criterion $\left(Q^{*}=Q\left(D^{2} / U_{0}^{2}\right)=100\right)$ coloured by velocity magnitude: Case 1 (top left), Case 2 (top right), Case 3 (bottom left) and Case 4 (bottom right).

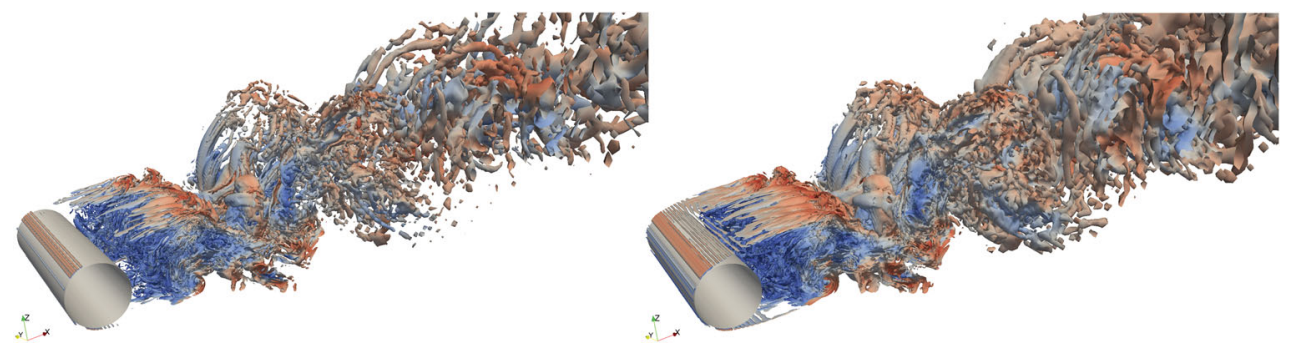

Figure 11. Iso contours of the $\sigma$ value coloured by the velocity magnitude for $\sigma=0.97$ (left) and $\sigma=$ 0.985 (right) for the Case 4.

are mode developed in case 4 and that the separated shear layer transition occurs earlier in that case. The same worm-like vortices are observed in Figure 11 which display the iso-value of $\sigma=0.97$ (Case 1 value) and $\sigma=0.985$ (Case 2 value) for the case 4 . The $\sigma$ parameter tends to decrease down to 0.5 or lower values for a few grid points in areas where dissipation is needed to ensure a good representation of physical phenomena or stays at values close to 1 when no dissipation is needed.

A last quality criterion may be obtained analysing the frequency content of the wake. This is done looking at the frequency spectrum of both streamwise and normal velocity fluctuations in the wake at $x / D=3$, see Figures 12 and 13, respectively. Power spectra are computed using 3 sequences of 10 vortex shedding cycles with $50 \%$ of overlapping using the periodogram technique of Welch [64] and Hanning window. It is seen that the main frequency peak is recovered in all cases, showing that the vortex shedding frequency $f_{v s}$ is accurately predicted. More interestingly, the existence of a secondary peak at a frequency 


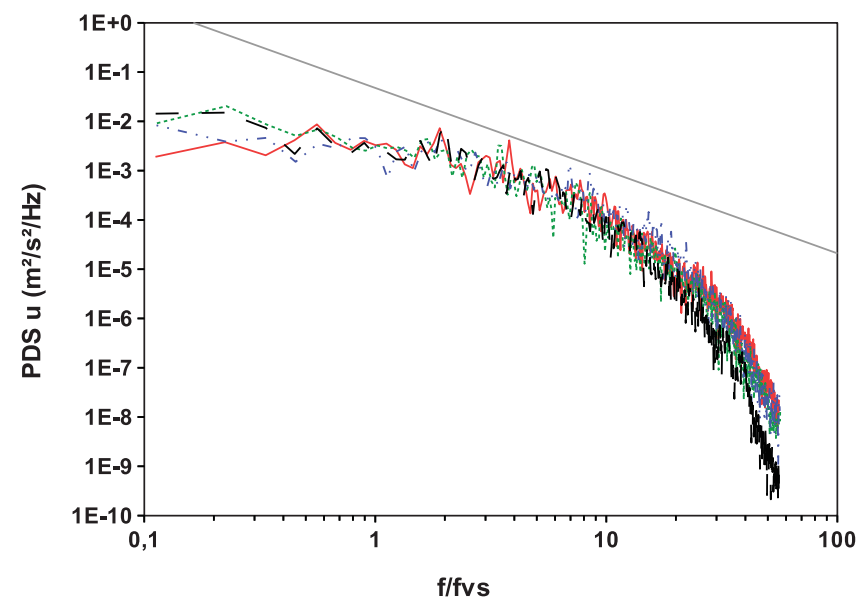

Figure 12. Power spectra density of the streamwise velocity at $x=3 D$ : present case $1-\quad$, present case $2 \ldots \ldots \ldots$, present case $3{ }_{n=-\ldots=\ldots}$, present case 4 _

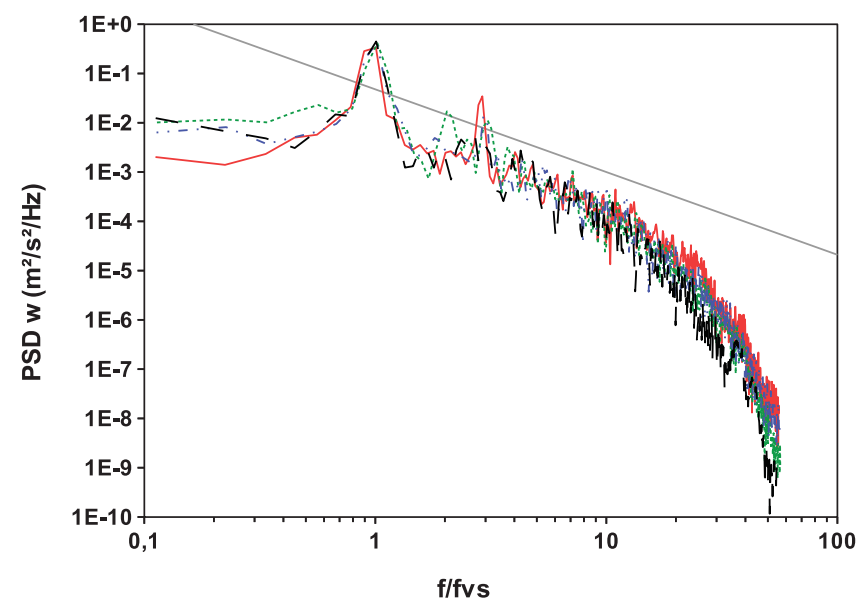

Figure 13. Power spectra density of the normal velocity at $x=3 D$ : present case $1 \ldots$ $2 \ldots \ldots \ldots$, present case $3{ }_{-\ldots-\ldots-\ldots}$, present case 4

three times larger than the primary peak, i.e. $f=3 f_{v s}$ in the normal velocity spectrum is accurately captured in case 4 , showing the very good quality of this simulation [49]. An inertial range with a slope is observed in all cases, whose width is about 1 decade, as in high-resolution LES and experiments presented in Ref. [49].

\section{Concluding remarks}

A new regularised collision model for Lattice Boltzmann-based LES of weakly compressible flows, referred to as the HRR-BGK collision model, has been presented. It relies on the modification of previously existing recursive regularised BGK models, which consists of hybridising the computation of the velocity gradient with a centred finite difference evaluation when reconstructing the regularised non-equilibrium part of the distribution 
functions. The resulting effect is the introduction of a stabilising hyperviscosity term, whose amplitude can be explicitly tuned via a control parameter $\sigma$. The resulting model can be used in both LES and ILES modes.

The new collision model has been assessed considering the flow around a cylinder at $\operatorname{Re}=3900$. The results obtained with a fixed uniform value of $\sigma$ exhibit a delay in the transition of the separated shear layers, a phenomenon which is also observed when an explicit subgrid viscosity term is added. On the opposite, very satisfactory results are obtained when using the dynamic version of the HRR-BGK collision kernel equipped with the Vreman's subgrid model. It is important noting that ILES based on the dynamic HRR-BGK is not strictly equivalent to a classical BGK coupled to the Vreman's model, since the scale-byscale distributions of the total dissipation are not equivalent. While the original Vreman model is associated to a classical Laplacian-based dissipation, the present ILES method introduces an bi-Laplacian-based dissipation. The use of higher order dissipation is known to preserve large-scale and inertial-range dynamics $[65,66]$, and has been successfully used in several ILES methods for Navier-Stokes equations, e.g. Refs. [67-73], with a few existing extensions to the LBM framework [74-77].

The significant differences between the dynamic version of the present ILES method and non-dynamic ones and also the classical explicit LES are due to the fact that most key features of the flow are governed by the transition in separated shear layers. This phenomenon is very sensitive to viscous and hyperviscous damping. A fully turbulent flow is less sensitive to fine details of the dissipative mechanisms, and much smaller differences would certainly occur in such a flow, as observed in ILES results based on Navier-Stokes equations.

\section{Acknowledgments}

This work was carried out using the ProLB solver. Professor Eric Lamballais is warmly acknowledged for providing experimental data.

\section{Disclosure statement}

No potential conflict of interest was reported by the authors.

\section{Funding}

This work was supported by the French project CLIMB, with the financial support of BPIFrance (Project No. P3543-24000), in the framework of the program 'Investissement d'Avenir: Calcul Intensif et Simulation Numérique'. This work was performed using HPC resources from GENCITGCC/CINES (Grant 2017-A0012A07679).

\section{ORCID}

Jérôme Jacob (D) http://orcid.org/0000-0001-9287-4167

Orestis Malaspinas (iD) http://orcid.org/0000-0001-9427-6849

Pierre Sagaut (D) http://orcid.org/0000-0002-3785-120X

\section{References}

[1] Garnier E, Adams N, Sagaut P. Large-eddy simulation for compressible flows. Berlin: Springer; 2009. 
[2] Sagaut P. Large eddy simulation for incompressible flows: an introduction. Berlin: Springer; 2005.

[3] Sagaut P, Deck S, Terracol M. Multiscale and multiresolution approaches in turbulence. London: Imperial College Press; 2006.

[4] Meyers J, Sagaut P. Is plane channel flow a friendly case for testing of large-eddy simulation subgrid-scale models?. Phys Fluids. 2007;19:048105.

[5] Meyers J, Sagaut P, Geurts BJ. Optimal model parameters for multi-obective large-eddy simulations. Phys Fluids. 2006;18:095103.

[6] Meyers J, Sagaut P, Geurts BJ. A computational error assessment of central finite-volume discretizations in large-eddy simulation using a Smagorinsky model. J Comput Phys. 2007;227(1):156-173.

[7] Grinstein FF, Margolin LG, Rider WJ. Implicit large eddy simulation: computing turbulent fluid dynamics. Cambridge: Cambridge University Press; 2007.

[8] Chen S, Doolen GD. Lattice Boltzmann method for fluid flows. Annu Rev Fluid Mech. 1998;30(1):329-364.

[9] Guo Z, Shu C. The lattice Boltzmann method and its applications in engineering. Singapore: World Scientific; 2013.

[10] Kruüger T, Kusumaatmaja H, Kuzmin A. The lattice Boltzmann method. Principles and practice. Berlin: Springer; 2017.

[11] Shan X, Yuan X-F, Chen H. Kinetic theory representation of hydrodynamics: a way beyond the Navier-Stokes equation. J Fluid Mech. 2006;550:413-441.

[12] Succi S. The lattice Boltzmann equation for fluid dynamics and beyond. Oxford: Oxford University Press; 2001.

[13] Ansumali S, Karlin IV, Succi S. Kinetic theory of turbulence modeling: smallness parameter, scaling and microscopic derivation of Smagorinsky model. Physica A. 2004;338(3-4): 379-394.

[14] Chen H, Kandasamy S, Orszag S, et al. Extended Boltzmann kinetic equation for turbulent flows. Science. 2003;301(5633):633-636.

[15] Chen H, Orszag SA, Staroselsky I, et al. Expanded analogy between Boltzmann kinetic theory of fluids and turbulence. J Fluid Mech. 2004;519:301-314.

[16] Chen H, Succi S, Orszag S. Analysis of subgrid scale turbulence using the Boltzmann Bhatnagar-Gross-Krook kinetic equation. Phys Rev E. Mar 1999;59(3):R2527-R2530.

[17] Chen S. A large-eddy-based lattice Boltzmann model for turbulent flow simulation. Appl Math Comput. 2009;215(2):591-598.

[18] Eggels JGM. Direct and large-eddy simulation of turbulent fluid flow using the latticeBoltzmann scheme. Int J Heat Fluid Flow. 1996;17(3):307-323.

[19] Filippova O, Succi S, Mazzocco F, et al. Multiscale lattice Boltzmann schemes with turbulence modeling. J Comput Phys. 2001;170(2):812-829.

[20] Girimaji SS. Boltzmann kinetic equation for filtered fluid turbulence. Phys Rev Lett. 2007;99(3):034501.

[21] Hou S, Sterling J, Chen S, et al. A lattice Boltzmann subgrid model for high Reynolds number flows. Fields Inst Comm. 1996;6:151-66.

[22] Krafczyk M, Tölke J, Luo L-S. Large-eddy simulations with a multiple-relaxation-time LBE model. Int J Mod Phys B. 2003;17:33-39.

[23] Malaspinas O, Sagaut P. Consistent subgrid scale modelling for lattice Boltzmann methods. J Fluid Mech. JUN 2012;700:514-542.

[24] Premnath KN, Pattison MJ, Banerjee S. Dynamic subgrid scale modeling of turbulent flows using lattice-Boltzmann method. Physica A. 2009;388(13):2640-2658.

[25] Teixeira CM. Incorporating turbulence models into the lattice-Boltzmann method. Int J Modern Phys C. 1999;9:1159-1175.

[26] Weickert M, Teike G, Schmidt O, et al. Investigation of the LES WALE turbulence model within the lattice Boltzmann framework. Comput Math Appl. 2010;59(7):2200-2214. Mesoscopic Methods in Engineering and Science, International Conferences on Mesoscopic Methods in Engineering and Science. 
[27] Bösch F, Chikatamarla SS, Karlin IV. Entropic multi-relaxation models for simulation of fluid turbulence. ESAIM: Proc Surveys. 2015;52:1-24.

[28] Bösch F, Chikatamarla SS, Karlin IV. Entropic multi-relaxation models for turbulent flows. Phys Rev E. 2015;92:43309.

[29] Di Ilio G, Dorschner B, Bella G, et al. Simulation of turbulent flows with the entropic multirelaxation time lattice Boltzmann method on body-fitted meshes. J Fluid Mech. 2018;849: 35-56.

[30] Dorschner B, Chikatamarla SS, Karlin IV. Transitional flows with the entropic lattice Boltzmann method. J Fluid Mech. 2017;824:388-412.

[31] Geier M. De-aliasing and stabilization formalism of the cascaded lattice Boltzmann automaton for under-resolved high Reynolds number flow. Int J Numer Methods Fluids. 2008;56:1249-1254.

[32] Geier M, Greiner A, Korvink JG. Cascaded digital lattice Boltzmann automata for high Reynolds number flow. Phys Rev E. 2006;73:066705.

[33] Geller S, Uphoff S, Krafczyk M. Turbulent jet computations based on MRT and cascaded lattice Boltzmann models. Comput Math Appl. 2013;65:1956-1966.

[34] Coreixas C, Wissocq G, Puigt G, et al. Recursive regularization step for high-order lattice Boltzmann methods. Phys Rev E. 2017;96:033306.

[35] Latt J. Hydrodynamic limit of lattice Boltzmann equations. PhD dissertation, University of Geneva, Geneva, Switzerland, 2007.

[36] Latt J, Chopard B. Lattice Boltzmann method with regularized pre-collision distribution functions. Math Comp Sim. 2006;72:165-168.

[37] Malaspinas O. Increasing stability and accuracy of the lattice Boltzmann scheme: recursivity and regularization. arXiv, pages 1-31, 2015

[38] Mattila KK, Philippi PC, HegeleJr LA. High-order regularization in lattice-Boltzmann equations. Phys Fluids. 2017;29(4):046103.

[39] Montessori A, Falcucci G, Prestininzi P, et al. Regularized lattice Bhatnagar-Gross-Krook model for two- and three-dimensional cavity flow simulations. Phys Rev E. 2014;89: 053317.

[40] Chapman S, Cowling TG. The mathematical theory of nonuniform gases. Cambridge: Cambridge University Press; 1960.

[41] Shan X. The mathematical structure of the lattices of the lattice Boltzmann method. J Comput Sci. 2016;17:475-481.

[42] Menter FR, Egorov Y. The scale-adaptive simulation method for unsteady turbulent flow predictions. Part 1: theory and model description. Flow Turbul Combust. 2010;85:113-138.

[43] Geurts BJ, Frölich J. A framework for predicting accuracy limitations in large-eddy simulation. Phys Fluids. 2002;14:L41-L44.

[44] Vreman AW. An eddy-viscosity subgrid-scale model for turbulent shear flow: algebraic theory and applications. Phys Fluids. 2004;16:3670-3681.

[45] Silvis MH, Remmerswaal RA, Verstappen R. Physical consistency of subgrid-scale model for large-eddy simulation of incompressible turbulent flows. Phys Fluids. 2017;29:015106.

[46] Trias FX, Folch D, Gorobets A, et al. Building proper invariants for eddy-viscosity subgrid-scale models. Phys Fluids. 2015;27:065103.

[47] Poinsot TJ, Lele SK. Boundary conditions for direct simulations of compressible viscous flows. J Comput Phys. 1992;101(1):104-129.

[48] Breuer M. Numerical and modeling influences on large eddy simulations for the flow past a circular cylinder. Int J Heat Fluid Flow. 1998;19(5):512-521.

[49] Parnaudeau P, Carlier J, Heitz D, et al. Experimental and numerical studies of the flow over a circular cylinder at Reynolds number 3900. Phys Fuids. 2008;20(8):085101.

[50] Alkishriwi N, Meinke M, Schröder W. A large-eddy simulation method for low Mach number flows using preconditioning and multigrid. Comput Fluids. 2006;35(10):1126-1136.

[51] Ouvrard H, Koobus B, Dervieux A, et al. Classical and variational multiscale LES of the flow around a circular cylinder on unstructured grids. Comput Fluids. 2010;39(7):1083-1094. 
[52] Dong S, Karniadakis GE, Ekmekci A, et al. A combined direct numerical simulation-particle image velocimetry study of the turbulent near wake. J Fluid Mech. 2006;569:185-207.

[53] Ma X, Karamanos G-S, Karniadakis GE. Dynamics and low-dimensionality of a turbulent near wake. J Fluid Mech. 2000;410:29-65.

[54] Alemi M, Pêgo JP, Maia R. Numerical investigation of the flow behavior around a single cylinder using large Eddy simulation model. Ocean Eng. 2017;145:464-478.

[55] Mani A, Moin P, Wang M. Computational study of optical distortions by separated shear layers and turbulent wakes. J Fluid Mech. 2009;625:273-298.

[56] Franke J, Frank W. Large eddy simulation of the flow past a circular cylinder at $\mathrm{ReD}=3900$. J Wind Eng Ind Aerodyn. 2002;90(10):1191-1206. 3rd European-African Conference on Wind Engineering.

[57] Kravchenko AG, Moin P. Numerical studies of flow over a circular cylinder at $\mathrm{ReD}=3900$. Phys Fluids. 2000;12(2):403-417.

[58] Lysenko DA, Ertesvåg IS, Rian KE. Large-eddy simulation of the flow over a circular cylinder at Reynolds number 3900 using the OpenFOAM toolbox. Flow Turbul Combust. 2012;89(4):491-518.

[59] Meyer M, Hickel S, Adams NA. Assessment of implicit large-eddy simulation with a conservative immersed interface method for turbulent cylinder flow. Int J Heat Fluid Flow. 2010;31(3):368-377. Sixth International Symposium on Turbulence and Shear Flow Phenomena.

[60] Abrahamsen Prsic M, Ong MC, Pettersen B, et al. Large eddy simulations of flow around a smooth circular cylinder in a uniform current in the subcritical flow regime. Ocean Eng. 2014;77:61-73.

[61] Wornom S, Ouvrard H, Salvetti MV, et al. Variational multiscale large-eddy simulations of the flow past a circular cylinder: Reynolds number effects. Comput Fluids. 2011;47(1):44-50.

[62] Zhang H, Yang J-M, Xiao L-F, Large-eddy simulation of the flow past both finite and infinite circular cylinders at $\operatorname{Re}=3900$. J Hydrodyn Ser B. 2015;27(2):195-203.

[63] D'Alessandro V, Montelpare S, Ricci R. Detached-eddy simulations of the flow over a cylinder at $\mathrm{Re}=3900$ using OpenFOAM. Comput Fluids. 2016;136:152-169.

[64] Welch P. The use of fast fourier transform for the estimation of power spectra: a method based on time averaging over short, modified periodograms. IEEE Trans Audio Electroacoust. Jun 1967;15(2):70-73.

[65] Borue V, Orszag SA. Forced three-dimensional homogeneous turbulence with hyperviscosity. Europhys Lett. 1995;29:687-692.

[66] Borue V, Orszag SA. Self-similar decay of three-dimensional homogeneous turbulence with hyperviscosity. Phys Rev E. 1995;51:R856-R859.

[67] Berland J, Lafon P, Daude F, et al. Filter shape dependence and effective scale separation in large-eddy simulations based on relaxation filtering. Comput Fluids. 2011;47:65-74.

[68] Daude F, Berland J, Emmert T, et al. A high-order finite-difference algorithm for direct computation of aerodynamic sound. Comput Fluids. 2012;61:46-63.

[69] Hickel S, Adams NA. On implicit subgrid-scale modeling in wall-bounded flows. Phys Fluids. 2007;19:105106.

[70] Hickel S, Adams NA, Domaradzki JA. An adaptive local deconvolution method for implicit LES. J Comput Phys. 2006;213:413-436.

[71] Stolz S, Adams NA. An approximate deconvolution procedure for large-eddy simulation. Phys Fluids. 1999;11(7):1699-1701.

[72] Stolz S, Adams NA, Kleiser L. An approximate deconvolution model for large-eddy simulation with application to incompressible wall-bounded flows. Phys Fluids. 2001;13(4):997-1015.

[73] Stolz S, Adams NA, Kleiser L. The approximate deconvolution model for large-eddy simulations of compressible flows and its application to shock-turbulent-boundary-layer interaction. Phys Fluids. 2001;13(10):2985-3001.

[74] Malaspinas O, Chopard B, Latt J. General regularized boundary condition for multi-speed lattice Boltzmann models. Comput Fluids. 2011;49(1):29-35. 
[75] Marié S, Gloerfelt X. Adaptive filtering for the lattice Boltzmann method. J Comput Phys. 2017;333:212-226.

[76] Ricot D, Marié S, Sagaut P, et al. Lattice Boltzmann method with selective viscosity filter. J Comput Phys. 2009;228(12):4478-4490.

[77] Sagaut P. Toward advanced subgrid models for Lattice-Boltzmann-based large-eddy simulation: theoretical formulations. Comput Math Appl. 2010;59(7):2194-2199. Mesoscopic Methods in Engineering and Science, International Conference on Mesoscopic Methods in Engineering and Science. 